\title{
Estrategias de gestión ambiental en las Instituciones de Educación Superior. El caso del Centro Universitario de los Valles
}

\section{Environmental management strategies in Institutions of Higher Education. The case of the University Center of the Valleys}

\author{
ACEVES-MÁRQUEZ, Martha Patricia†, AYALA-RAMIREZ, Suhey, CASTILLO-GIRON, Víctor \\ Manuel y MEDINA-CELIS, Laura Margarita
}

Universidad de Guadalajara - Centro Universitario de Ciencias Económico Administrativas

ID 1er Autor: Martha Patricia, Aceves-Márquez / ORC ID: 0000-0002-8840-0513, CVU CONACYT ID: 739031

ID 1er Coautor: Suhey, Ayala-Ramirez / ORC ID: 0000-0003-1079-9605, CVU CONACYT ID: 171341

ID 2do Coautor: Víctor Manuel, Castillo-Giron / ORC ID: 0000-0002-8307-2952, CVU CONACYT ID: 31156

ID3er Coautor: Laura Margarita, Medina-Celis / ORC ID: 0000-0002-3696-7243, CVU CONACYT ID: 328481

DOI: $10.35429 / J U S D .2019 .14 .5 .31 .41$

Recibido: 10 de Enero, 2019; Aceptado 30 Marzo, 2019

\section{Resumen}

La gestión ambiental del agua forma parte de los objetivos de desarrollo sostenible y su importancia radica en ser un elemento esencial para la vida. Siendo las Instituciones de Educación Superior (IES) un ente con un papel relevante y su labor impacta en dos vertientes: en el actuar operativo y en la manera de actuar de los individuos que la conforman. El objetivo de este artículo es analizar las estrategias que movilizan las IES para consolidarse como un campus sustentable y que puedan ser aplicables a otras instituciones, tomando como caso de estudio el Centro Universitario de los Valles de la Universidad de Guadalajara. La metodología se basó en un marco internacional que toma en cuenta diversos ejes donde las IES intervienen, para esto se utilizó la "Encuesta para el Diagnóstico de la Institucionalización del Compromiso Ambiental de las Universidades Colombianas", la información se complementó y valido mediante la consulta de los sitios webs institucionales, plataformas, informes, gacetas y entrevistas (Münch y Ángeles, 2015). La investigación pretende generar estrategias, para el buen uso y manejo del recurso hídrico en las IES impactando sus ejes sustantivos, para asegurar el consumo responsable y fomentar la cultura ambiental en la comunidad universitaria.

Estrategias, Gestión ambiental del agua, IES

\begin{abstract}
The environmental management of water is part of the objectives of sustainable development and its importance lies in being an essential element for life. Being the Institutions of Higher Education (HEIs) a work with a relevant role and its work impacts in two aspects: in the operative action and in the way of acting of the individuals that make it up. The objective of this article is to analyze the strategies that the HEIs mobilize to consolidate as a sustainable campus and that may be applicable to other institutions, taking the taking the University Center of the Valleys of the University of Guadalajara as a case study. The methodology was based on an international framework that takes into account several axes where HEIs intervene, for this the "Survey for the Diagnosis of the Institutionalization of the Environmental Commitment of Colombian Universities" was used, the information was supplemented and validated through consultation of institutional websites, platforms, reports, gazettes and interviews (Münch and Ángeles, 2015). The research aims to generate strategies for the proper use and management of water resources in HEIs impacting their substantive axes, to ensure responsible consumption and promote environmental culture in the university community.
\end{abstract}

Strategies, Environmental water management, IES

Citación: ACEVES-MÁRQUEZ, Martha Patricia, AYALA-RAMIREZ, Suhey, CASTILLO-GIRON, Víctor Manuel y MEDINA-CELIS, Laura Margarita. Estrategias de gestión ambiental en las Instituciones de Educación Superior. El caso del Centro Universitario de los Valles. Revista del Desarrollo Urbano y Sustentable. 2019. 5-14: 31-41

\footnotetext{
$\dagger$ Investigador contribuyendo como primer Autor
} 


\section{Introducción}

Dentro de los objetivos del Desarrollo Sostenible se encuentra el garantizar la disponibilidad y la gestión sostenible del agua y el saneamiento para todos, la importancia del recurso hídrico radica en que es fuente de vida de los ecosistemas naturales y donde exista vida humana, estos últimos incluyen a las ciudades que a su vez están conformadas por instituciones que brindan servicios y uno de ellos es el sector educativo. Las universidades se rigen bajo principios y valores vinculados a las prácticas y conductas que tienen los individuos que las conforman: directivos, docentes y estudiantes que generan un valor positivo o negativo dependiendo del nivel de compromiso con el medio ambiente y la sustentabilidad ambiental. Teniendo como marco de referencia la sustentabilidad, ésta marca las directrices para el cuidado y buen uso del agua considerando un aprovechamiento de los recursos de manera eficiente sin comprometer las necesidades de las futuras generaciones, para satisfacer sus propias necesidades (CMMAD, 1987). Es ahí donde surgen nuevos enfoques que marcan el rumbo de las instituciones generando un valor agregado con este nuevo modelo que destaca la importancia del medio ambiente en todas las dimensiones que las universidades tienen relación.

Cabe señalar que las universidades trabajan bajo regímenes que se desprenden de acuerdos internacionales y que van permeando de manera vertical hasta llegar a todos los sectores. De esta manera, las universidades involucran ejes centrales estipulados en el Plan de Desarrollo Institucional, que incluye la docencia, extensión, investigación y gestión operativa/ infraestructura. En México la gestión ambiental del agua se ha limitado al abastecimiento de la misma, sobre todo para las actividades económicas y consumo humano; sin embargo en el afán de no sufrir escasez de agua, ha dado pie a macroconstrucciones como las presas para abastecimiento de la industria, sobreexplotación de pozos subterráneos, que han traído consecuencias como la desaparición o migración de comunidades enteras, riqueza forestal y cultural, además de la pérdida de especies endémicas, por falta de una visión que genere soluciones sustentables viables, para evitar externalidades negativas ${ }^{1}$ a terceros.

\footnotetext{
${ }^{1}$ Es una acción que afecta negativamente a terceros, sin que estos sean los responsables de dicha acción.

Dichas externalidades afectan principalmente a comunidades rurales ya que es ahí, donde se encuentra esta riqueza natural, y que son detectadas rápidamente cuando las ciudades empiezan a colapsar debido a la falta de algún bien en este caso el agua, o al menos ha sido el justificante más aceptado por la sociedad en aras del desarrollo.

En este sentido, la aportación de las universidades es relevante como una propuesta de solución desde su trinchera, dado que es aquí donde estudian los futuros líderes de opinión, ingenieros, arquitectos, gestores, administradores, entre otros cientos profesiones que darán respuesta a los problemas ambientales, sociales y económicos en el corto, mediano y largo plazo. Es decir, en su actuar cotidiano y lo que se espera en un futuro. Bajo este contexto, el objetivo del presente artículo es analizar las estrategias que movilizan las IES para consolidarse como un campus sustentable y que puedan ser aplicables a otras instituciones, tomando como caso de estudio el Centro Universitario de los Valles de la Universidad de Guadalajara.

Partimos de la hipótesis de que a través del diseño e implementación de estrategias de gestión ambiental de los recursos hídricos vinculadas estrechamente a las actividades sustantivas de las universidades como el diseño curricular, la investigación, la gestión operativa, y la sensibilización de la comunidad universitaria en la cultura del manejo y uso eficiente del agua, puede contribuir a la gestión integral del recurso hídrico cada vez más escaso, para la consolidación formal de Universidades a Universidades Sustentables.

Además de esta introducción, el artículo se estructura en tres partes. En la primera se hace un análisis teórico en relación al desarrollo sustentable, la responsabilidad social universitaria, y la gestión ambiental con enfoque en el recurso hídrico. El segundo apartado muestra los resultados más significativos de la investigación y la propuesta de las estrategias del estudio de caso. El tercero aborda las conclusiones de la investigación y algunas recomendaciones. 


\section{Descripción del método}

En algunos países del mundo existen métricas que reconocen las buenas prácticas de sustentabilidad, incluso son evaluadas anualmente esto con la finalidad de ratificar su compromiso, avances y nuevas metas, una de estos indicadores se encuentran en el ranking GreenMetric, sin embargo no existe paridad de condiciones ya que las características económicas, y culturales difieren siendo de mayor utilidad ajustarse a las condiciones que se tienen y a partir de ello avanzar al terreno de la sustentabilidad.

El instrumento que se utilizó para recabar parte de la información fue la "Encuesta para el Diagnóstico de la Institucionalización del Compromiso Ambiental de las Universidades Colombianas", dicha información se complementó y valido mediante la consulta de los sitios webs institucionales, plataformas, informes, reportes, gacetas $\mathrm{y}$ entrevistas (Münch y Ángeles, 2015).

Para el análisis del estudio de caso se optó por el Centro Universitario de los Valles (CUValles), un centro regional que forma parte de la Red Universitaria de Jalisco, distinguido por sus buenas prácticas de sustentabilidad, galardonado un par de veces por sus méritos en el terreno ambiental (Premio al Mérito Ecológico) y social (Centro Incluyente), siendo un detonador para la región donde se desarrolla y que ha contemplado en su Plan Maestro a la sustentabilidad, así como en sus actividades científicas y académicas. Siendo un caso de éxito en comparativa con otros centros de la red universitaria de Jalisco, con impacto en los ejes donde tiene concurrencia.

\section{Marco de referencia}

\section{Desarrollo sustentable}

Uno de los conceptos que sustentan el campo de la gestión ambiental es el de Desarrollo sustentable, que si bien, éste abarca también las dimensiones de la economía y la sociedad, las cuestiones ambientales son un pilar importante y proyecta un equilibrio entre todas las esferas, dando pie al desarrollo sustentable.
En este sentido, es relevante recuperar la dimensión ambiental del concepto de desarrollo sustentable que tiene como objetivo la protección, cuidado y uso adecuado de los recursos naturales, particularmente el recurso hídrico, que forma parte de los ciclos naturales de todos los ecosistemas y que se ha visto interrumpido por las actividades antropogénicas.

Antes de continuar con la definición de desarrollo sustentable vale la pena esclarecer la confusión entre las personas de habla hispana sobre el conocimiento de los términos de desarrollo sustentable y desarrollo sostenible, según Coen (2006), aunque en morfología y lexicología ambas comparten las mismas raíces etimológicas (sostener, sustentar y mantener) la diferencia de estas acepciones es la separación de los verbos ya que sostenible refiere a un aspecto de permanecer firmemente, inalterable, inamovible, mientras que sustentable implica un proceso continuo, "lo que requiere que se esté alimentando, proporcionando los medios de sobrevivencia y de persistencia" [(Coen, 2006) citado en Zapata et al, 2016].

Cabe destacar que teóricamente se ha hecho uso indiscriminado de ambos conceptos incluso se utiliza como sinónimo o errores de traducción, incluso son pocos los autores que se detienen a esclarecer de manera puntual el concepto, en este sentido cabe mencionar que en el caso de algunas citas textuales se optó por dejar el concepto de desarrollo sostenible, ya que no se pretende alterar los textos ante la idea de focalizar todo el documento al desarrollo sustentable; no obstante en el caso de redacción propia, fue preferible hacer uso del concepto de desarrollo sustentable y sustentabilidad.

La Asociación Española de Contabilidad y Administración de Empresas (AECA) define al Desarrollo sustentable como: "Un proceso de largo plazo generador de progreso económico, social, cultural, político y medioambiental para las comunidades humanas. Es decir, un desarrollo polivalente de carácter integral" (2006). De esta acepción, aplicado a una organización, considera una visión que tiene en cuenta la triple dimensión económica, social y medioambiental. Cabe destacar que, en el referente anglosajón, centra la atención en el carácter marcadamente medioambiental, refiriéndose al uso racional de los recursos naturales que evite el deterioro del medio ambiente.

ACEVES-MÁRQUEZ, Martha Patricia, AYALA-RAMIREZ, Suhey, CASTILLO-GIRON, Víctor Manuel y MEDINA-CELIS, Laura Margarita. Estrategias de gestión ambiental en las Instituciones de Educación Superior. El caso del Centro Universitario de los Valles. Revista del Desarrollo Urbano y Sustentable. 2019 
De ahí que, desarrollo sostenible y sostenibilidad sean términos utilizados por compañías industriales que suelen influir en el entorno natural (AECA, 2006).

Uno de los conceptos más reconocidos a nivel mundial es el que se dio a conocer en el Informe Brundtland en 1987, el cual describe al desarrollo sustentable como:

\section{"El desarrollo que satisface las necesidades del presente sin comprometer las necesidades de las futuras generaciones para satisfacer sus propias necesidades" (Brundtland, 1987)}

Dicho concepto pretendía ser, en un principio, rector en el actuar de la sociedad que impactará de manera positiva en el terreno medioambiental, al mismo tiempo exige cambios urgentes en los sistemas económicos y la dinámica poblacional (patrones de consumo, factores demográficos) con el objetivo de transitar a una cultura sensible y responsable con la naturaleza que involucre el estudio de los ecosistemas naturales, así como su capacidad de carga y de resiliencia.

De manera particular se puede robustecer este concepto integrando otros factores, quedando de la siguiente manera: Conjunto de procesos evolutivos, que potencializa el avance científico en beneficio individual y social, en el ámbito político, cultural, económico, tecnológico y ambiental para satisfacer las necesidades del presente y mejorar las condiciones de vida, sin afectar las necesidades de las futuras generaciones y la capacidad de carga de los ecosistemas naturales incluidos: el agua, el aire, el suelo y la biodiversidad.

A escala internacional se han firmado un sinfín de tratados y acuerdos internacionales que tienen estrecha relación con el desarrollo sustentable y que han servido como marco de referencia para los planes y programas de desarrollo que permean a nivel nacional, estatal y local. Dentro de estos acuerdos destacan:

Agenda 2030 para el Desarrollo Sostenible, el cual consta de 17 objetivos que abarcan la esfera económica, social y ambiental, que para fines de la investigación se pone en manifiesto el objetivo 6 "Agua limpia y saneamiento" que tiene el reto de:
- Garantizar la disponibilidad de agua, su gestión sostenible y el saneamiento para todos.

- Lograr el acceso universal y equitativo del agua potable.

- Mejorar la calidad del agua mediante la reducción de la contaminación por descargas de productos químicos peligrosos.

Reducir el porcentaje de aguas residuales sin tratar.

Aumentar sustancialmente el reciclado y reutilización a través del uso de las tecnologías.

- Cooperar activamente entre países para la creación de programas relativos con el agua, incluido el acopio y almacenamiento de agua (ONU, 2015).

\section{Responsabilidad Social Universitaria}

Otro concepto que tiene una estrecha relación con la protección ambiental es la Responsabilidad Social Universitaria, según Carroll (1979) menciona que la RS es el conjunto de expectativas económicas, legales, éticas y discrecionales de la sociedad con respecto a las organizaciones en un momento dado del tiempo.

La Responsabilidad Social no es un modelo exclusivo de la empresa, y actualmente algunas universidades han adoptado prácticas en concordancia con la RS, dando pie al concepto de Responsabilidad Social Universitaria (RSU), cabe señalar que la definición comenzó a consolidarse a principios del 2000, con los esfuerzos teóricos y prácticos de la Red Chilena, Universidad Construye País y la Red Latinoamericana de Universidades que actualmente no están activas, sin embargo, sentaron las bases para la construcción de la definición.

La Red Universidad Construye País define la RSU así:

"Entendemos por Responsabilidad Social Universitaria la capacidad que tiene la universidad de difundir y poner en práctica un conjunto de principios y valores generales y específicos, por medio de cuatro procesos clave: gestión, docencia, investigación y extensión. Así asume su responsabilidad social ante la propia comunidad universitaria y el país donde está inserta" [(Jiménez, 2002) citado en Vallaeys, 2019].

ACEVES-MÁRQUEZ, Martha Patricia, AYALA-RAMIREZ, Suhey, CASTILLO-GIRON, Víctor Manuel y MEDINA-CELIS, Laura Margarita. Estrategias de gestión ambiental en las Instituciones de Educación Superior. El caso del Centro Universitario de los Valles. Revista del Desarrollo Urbano y Sustentable. 2019 
Otra definición de RSU, promovida por la Iniciativa de Ética, Capital Social y Desarrollo del Banco Interamericano de Desarrollo (BID), basada en buenas prácticas aplicables a los procesos de formación, investigación, extensión y gestión administrativa; a partir de esto, Vallaeys (2006) define el concepto como:

"Una política de gestión de calidad ética de la Universidad que busca alinear sus cuatro procesos (gestión, docencia, investigación y extensión) con la misión universitaria, sus valores y compromiso social, mediante el logro de la congruencia institucional, la transparencia y la participación de toda la comunidad universitaria (autoridades, estudiantes, docentes, administrativos) con los múltiples actores sociales interesados en el buen desempeño universitarios y necesitados de él, para la transformación efectiva de la sociedad hacia la solución de sus problemas de exclusión, inequidad y sostenibilidad"

[(Vallaeys, 2006) citado en Vallaeys, 2019].

Cabe destacar que, en ambas definiciones la labor universitaria no se centra solamente en las actividades académicas, sino que integra la tarea administrativa que enmarca diversas actividades como: compras, gobierno, planificación, prestación de servicios, matrícula, marketing, selección de personal. Por otro lado, el eje de extensión no trata de crear escenarios para transmitir información a la sociedad de una manera pasiva, sino que deben existir ambientes para la participación activa que involucre a la comunidad en la búsqueda de la resolución de conflictos [(Appe et al., 2017), citado en Vallaeys, 2019].

La RSU busca desarrollar el conocer, el trabajar y actuar para cuidar y preservar el entorno ambiental sustentable y de forma inmediata evitar daños y alcanzar beneficios dentro de la comunidad universitaria, motivar la responsabilidad social universitaria para tomar conciencia de los problemas institucionales, pero también comunitarios para lograr establecer la mejora continua y la calidad del medio ambiente de todos los universitarios y sus familias (Castillo et al, 2018).

\section{Gestión del Agua}

A la par de estos conceptos existen otros referentes teóricos que abordan específicamente temas de agua mediante el concepto de gestión del agua o de los recursos hídricos, según la Comisión Nacional del Agua (CONAGUA) en México, lo define como el proceso que promueve el manejo y desarrollo coordinado del agua, con el fin de maximizar el bienestar social y económico equitativamente sin comprometer la sustentabilidad de los ecosistemas vitales. Dicha gestión está íntimamente vinculada con el desarrollo sustentable (CONAGUA, 2017).

\section{Para Tiburcio Sánchez y}

Perevochtchikova (2012) la gestión aplicada al recurso hídrico se enfoca en la buena administración del agua en términos de suministro de agua potable, calidad, saneamiento, control de inundaciones, conservación de ecosistemas acuáticos y solución de conflictos generados por la demanda del recurso para actividades de tipo productivo y uso doméstico. Desde el punto de vista jurídico la gestión del agua en México, se define como el:

"Proceso sustentado en el conjunto de principios, políticas, actos, recursos, instrumentos, normas formales y no formales, bienes, recursos, derechos, atribuciones y responsabilidades, mediante el cual coordinadamente el Estado, los usuarios del agua y las organizaciones de la sociedad, promueven e instrumentan para lograr el desarrollo sustentable en beneficio de los seres humanos y su medio social, económico y ambiental, (1) el control y manejo del agua y las cuencas hidrológicas, incluyendo los acuíferos, por ende su distribución y administración, (2) la regulación de la explotación, uso o aprovechamiento del agua, y

(3) la preservación y sustentabilidad de los recursos hídricos en cantidad y calidad, considerando los riesgos ante la ocurrencia de fenómenos hidrometeorológicos extraordinarios y daños a ecosistemas vitales y al medio ambiente. La gestión del agua comprende en su totalidad a la administración gubernamental del agua". (Cámara de Diputados del H. Congreso de la Unión, 1992: artículo 3 fracción XXVIII). 
Estas definiciones coinciden con armonizar el uso adecuado, control y manejo del agua en cantidad (cobertura) y calidad, considerando las características de cada región hidrológica, así como la administración gubernamental del recurso; además considerar cuestiones como su tratamiento y descargas antes y después de la incorporación a las actividades antropológicas, tal como lo señalan los marcos internacionales. (Tiburcio Sánchez y Perevochtchikova, 2012), sin embargo, en México estos principios han sido laxos y flexibles ocasionando fragilidad a los ecosistemas acuáticos.

En este sentido el asunto del agua va más allá de las implicaciones técnicas y de distribución, éste tiene cabida en el ámbito económico, social, político, ambiental y cultural. Debido a que el recurso es esencial para la vida y la sustancia prima para las actividades antropogénicas en todos los sectores, primario, secundario y de servicios, que en suma demandan grandes volúmenes de agua (Chavarro Velandia, 2011).

La gestión del agua es competencia de todos los usuarios, y algunos actores han comenzado a hacer esfuerzos para su aprovechamiento sustentable, no obstante, permanece latente la problemática del agua en términos de calidad, disponibilidad, abastecimiento y sobreexplotación de acuíferos. Además, existen zonas en condiciones críticas, debido a la sobrepoblación y las actividades económicas que sostienen a esas regiones o complejos urbanos; agudizados por la escasa planeación de la infraestructura urbana y la baja inversión en la gestión integral del agua.

\section{Resultados}

Retomando la gestión ambiental en el actuar universitario, para el caso de docencia se realizó un análisis de la currícula de cada uno de los programas educativos de pregrado y posgrado del estudio de caso, y se encontró que 9 de los 18 programas educativos que se ofertan en el CUValles, cuenta con algún contenido temático en materia de medio ambiente lo que representa el 50 por ciento del total, siendo la Licenciatura en Educación con la más representativa en cuanto a contenido temático de las asignaturas especializantes en educación ambiental (CUValles, 2018).
Para el caso de posgrados solo 1 de 5 cuenta con tópicos de sustentabilidad, siendo el Programa de la Maestría en Estudios Socioterritoriales considerado de los más avanzados en la incorporación de la perspectiva de sustentabilidad sobre todo en la generación de tesis y productos de investigación.

En este sentido una estrategia para este eje es integrar una asignatura que abarque temas de sustentabilidad y problemática ambiental para que sea inculcada en la población estudiantil desde el primer semestre como tronco común, para forjar alumnos comprometidos con el medio ambiente que lleven a cabo buenas prácticas de sustentabilidad en su actuar cotidiano que permita el proceso de informacióncomprensión-participación ciudadana dentro y fuera del aula (Arrojo, S.F.).

Pudiendo combinar las actividades de docencia con actividades extracurriculares como diplomados, talleres y cursos que con tópicos selectos de medio ambiente (certificaciones, gestoría ambiental, auditorías ambientales), a continuación, se muestra la Tabla 1 que incluye los programas educativos que sirven de fomento a la cultura ambiental en los estudiantes del CUValles (2019a):

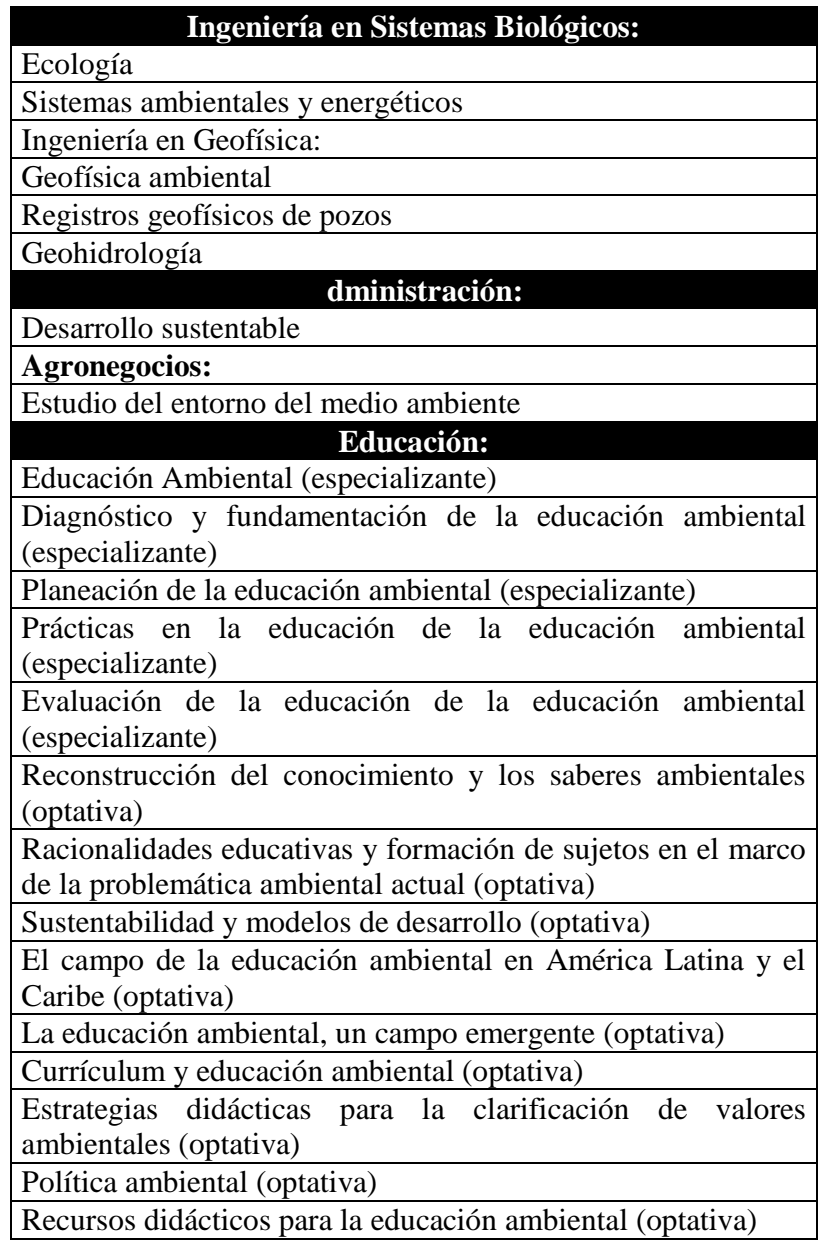

ACEVES-MÁRQUEZ, Martha Patricia, AYALA-RAMIREZ, Suhey, CASTILLO-GIRON, Víctor Manuel y MEDINA-CELIS, Laura Margarita. Estrategias de gestión ambiental en las Instituciones de Educación Superior. El caso del Centro Universitario de los Valles. Revista del Desarrollo Urbano y Sustentable. 2019 


\begin{tabular}{|l|}
\hline \multicolumn{1}{|c|}{ Ingeniería Mecatrónica: } \\
\hline Desarrollo sustentable (optativa abierta) \\
\hline Impacto ambiental de las obras de ingeniería (optativa abierta) \\
\hline \multicolumn{1}{c|}{ Nivelación de la Licenciatura en Enfermería: } \\
\hline Salud y sustentabilidad ambiental \\
\hline \multicolumn{1}{|c|}{ Licenciatura en Psicología: } \\
\hline Psicología Ambiental y Urbana \\
\hline Ciencia ambiental \\
\hline Medio ambiente y Desarrollo Sustentable \\
\hline Impacto ambiental en la sociedad \\
\hline $\begin{array}{l}\text { Proyectos de intervención social en el ámbito Ambiental I } \\
\text { (especializante selectiva) }\end{array}$ \\
\hline $\begin{array}{l}\text { Proyectos de intervención social en el ámbito Ambiental II } \\
\text { (especializante selectiva) }\end{array}$ \\
\hline \multicolumn{1}{c|}{ Maestría en Estudios Socio-territoriales: } \\
\hline Medio ambiente y Desarrollo Sustentable (optativa) \\
\hline
\end{tabular}

Tabla 1 Programas Educativos del CUValles que incluyen la formación ambiental y sustentabilidad en la currícula

Fuente: Elaboración Propia con base a CUValles (2019)

Otro eje de importancia dentro del ecosistema educativo son los grupos de investigación, formalmente constituidos como Cuerpos Académicos, de éstos se desprenden proyectos de investigación que dan solución a problemáticas sociales, ambientales y económicos siendo un campo de acción de las instituciones de educación superior que involucra a la comunidad universitaria: académicos y estudiantes.

En este sentido el CUValles (2019b) cuenta con el Cuerpo Académico: Energía y Medio Ambiente y el de Estudios Sustentables en las Organizaciones, que de manera directa promueven la cultura ambiental y son entes generadores de soluciones en colaboración con otros investigadores y alumnos de la Red Universitaria e incluso externos.

Algunas investigaciones se enfocan al estudio de la problemática a nivel local y regional destacando productos de investigación relacionados con la sustentabilidad de cuerpos de agua, la evaluación de la calidad del agua de cuencas importantes en el país como es la Cuenca-Lerma Chapala Santiago. En la Tabla 2. se describen las líneas de investigación con que cuenta el CUValles y el CA al que pertenecen.

\begin{tabular}{|l|l|l|}
\hline \multicolumn{2}{|c|}{$\begin{array}{l}\text { Lombre del Cuerpo } \\
\text { Académico }\end{array}$} & \multicolumn{2}{c|}{ Líneas de investigación } \\
\hline $\begin{array}{l}\text { CA UDG-CA-676 } \\
\text { Energía y Medio }\end{array}$ y $\begin{array}{l}\text { y/o 2: Estudios fisicoquímicos } \\
\text { Ambiente }\end{array}$ & $\begin{array}{l}\text { solucionar problemas ambientales } \\
\text { e industriales. }\end{array}$ \\
\hline $\begin{array}{l}\text { CA UDG-CA-678 } \\
\text { Estudios Sustentables en } \\
\text { las Organizaciones }\end{array}$ & $\begin{array}{l}\text { LGAC 2: Gestión de las áreas } \\
\text { funcionales para el desarrollo } \\
\text { sustentable en las organizaciones. }\end{array}$ \\
\hline
\end{tabular}

Tabla 2 CA y LGAC del CUValles relacionadas con temas de medio ambiente

Fuente: Elaboración propia con base a CUValles (2019b)

Si bien el Centro Universitario cuenta con dos líneas de investigación habría que contemplar el número de proyectos que impactan directamente en la gestión integral de los recursos hídricos para conocer el impacto real respecto a este recurso natural esencial para la vida. En la búsqueda de evidencias a través de la investigación documental se encontraron algunas investigaciones directamente relacionadas con el recurso hídrico y que son parte del acervo contenido en la Revista de Cultura, Tecnología y Patrimonio del mismo centro, las cuales se enlistan a continuación:

- $\quad$ Agua y agricultura en la Región LermaChapala de Michoacán (Número, 18. Año 2014).

Proyección estocástica del nivel de desarrollo sustentable de los municipios de Gómez Farías, Jalisco (Número, 11. Año 2011).

- Desarrollo sustentable utopías del siglo XX, competencia del siglo XXI. (Número, 10. Año 2010).

- La sustentabilidad de la cuenca del río Santiago y su relación con Guadalajara (Número, 7. Año 2009).

Turismo rural: el desarrollo local sustentable. Las fallas geológicas de Ameca y Chapala (Número, 1. Año 2006).

Respecto a los recursos financieros asignados no se tiene con certeza el monto asignado a cada proyecto de investigación, sin embargo, en 2017, el CUValles lanzó una convocatoria dirigida al impulso a la Investigación y Desarrollo Sobre el Agua y la Violencia en la Región de los Valles" que culminó en artículos de investigación científica (CUValles, 2017), dichas prácticas sirven de incentivos positivos de fomento a la nueva cultura del agua. A continuación, se muestran los títulos de las investigaciones realizadas: 


\begin{abstract}
- “Optimización de la energía por hidrógeno mediante sistemas biológicos para la remediación de agua".

"Diseño de una red de sitios para muestreo para la evaluación de la calidad del agua en la Presa La VegaCocula".

"El Estudio de Dilemas de Recursos Hídricos desde una Aproximación de la Psicología Experimental".
\end{abstract}

Dichos artículos serán la base para robustecer el acervo bibliográfico para los futuros especialistas de los programas educativos de Sistemas biológicos, Diseño molecular de materiales, Ciencias físico matemáticas, entre otras.

Respecto al eje de extensión ${ }^{2}$, el CUValles organiza eventos de fomento a la cultura ambiental, algunos de ellos en el marco del día mundial de los humedales, con la finalidad de contribuir a la solución de problemas de agua en comunidades, en colaboración con gobiernos locales y regionales, para ello se creó la Junta Intermunicipal de Medio Ambiente de Valles (JIMAV), donde se toman acciones para la restauración y conservación de cuerpos de agua y otros temas relacionados. Todo este conjunto de acciones hizo acreedor al Premio al Mérito Ecológico 2016. A continuación, se muestran otros eventos y acciones que forman parte de sus actividades de extensión:

- Creación de espacio para la capacitación en Sistemas acuícolas.

En el Marco Mundial de los Humedales, se llevaron a cabo actividades de educación ambiental para la preservación de humedales, en la comunidad aledaña a la Presa La Vega.

- En el Marco Mundial de los Humedales, se llevaron a cabo actividades de educación ambiental para la preservación de humedales, en la comunidad del municipio donde se ubica la Presa de Teuchitlán.

Se creó la Junta Intermunicipal de Medio Ambiente de Valles (JIMAV).

\footnotetext{
2 La extensión universitaria tiene como misión difundir las diversas manifestaciones del arte y la cultura nacional y universal, así como los conocimientos científicos, tecnológicos, y humanísticos que se desarrollan en la Universidad para enriquecer la formación de los alumnos, beneficiar lo más ampliamente posible a toda la sociedad mexicana, y fortalecer la identidad nacional.

ISSN 2414-4932

ECORFAN® Todos los derechos reservados
}

\begin{abstract}
- $\quad$ En el Marco del Día Mundial del Medio Ambiente, se impartieron conferencias relacionados con el manejo de residuos sólidos y el manejo integral del sitio Ramsar "Presa la Vega", dichas conferencias fueron impartidas por personal de la SEMADET.

En el Marco del Día Mundial de la Educación Ambiental se realizaron conferencias con temas relacionados a la sostenibilidad y se presentaron las acciones que se llevan a cabo en la Universidad de Guadalajara a nivel institucional.
\end{abstract}

En materia de difusión, se cuenta con "La gaceta del CUValles" que se publica de manera periódica información relevante de las acciones que lleva a cabo en la dimensión social y ambiental (CUValles, 2019c). Además de conferencias, ferias y foros que promueven la cultura ambiental, en concordancia con la misión del Centro, cuyo compromiso es la formación integral de líderes socialmente responsables. A través del cumplimiento de sus funciones sustantivas, responde a las necesidades locales, mediante al desarrollo de la cultura emprendedora, el respeto a la identidad regional y la generación y aplicación de nuevos conocimientos que incidan en el desarrollo sustentable de la región Valles.

Respecto a la gestión operativa ${ }^{3}$, este eje contempla aspectos de ahorro en agua, sanidad para consumo humano, riego y gestión de aguas residuales, en este sentido el CUValles ha tomado cartas en el asunto para minimizar su impacto ambiental implementando infraestructura para reducir su huella hídrica, con la instalación de dos planta de tratamiento de aguas residuales y Sistemas de Captación de Agua de Lluvia (SKALL ${ }^{4}$ ) en diversos edificios como la Rectoría, el gimnasio de usos múltiples y otros edificios, además de la sustitución de sanitarios con características de bajo consumo y grifos ecológicos (con temporizador), logrando impactar en la nueva cultura del cuidado del agua, estas acciones han sido un diferenciador con otras universidades a nivel estatal $y$ nacional.

\footnotetext{
${ }^{3}$ Se refiere a la infraestructura física y la forma de operar de las mismas

${ }^{4}$ Sistema de Captación de Agua de Lluvia, es una ecotecnia que se utiliza para dotar de agua potable en comunidades o viviendas, en cada uno de sus componentes con especificaciones técnicas requeridas, consiste en un diseño que permita interceptar, recolectar y almacenar el agua de lluvia. ACEVES-MÁRQUEZ, Martha Patricia, AYALA-RAMIREZ, Suhey, CASTILLO-GIRON, Víctor Manuel y MEDINA-CELIS, Laura Margarita. Estrategias de gestión ambiental en las Instituciones de Educación Superior. El caso del Centro Universitario de los Valles. Revista del Desarrollo Urbano y Sustentable. 2019
} 
De esta manera el CUValles se ha posicionado bajo el concepto de Campus Sustentable- Campus Responsable. A continuación, se muestra un compendio de imágenes que dan muestra de las practicas que realiza el CUValles.

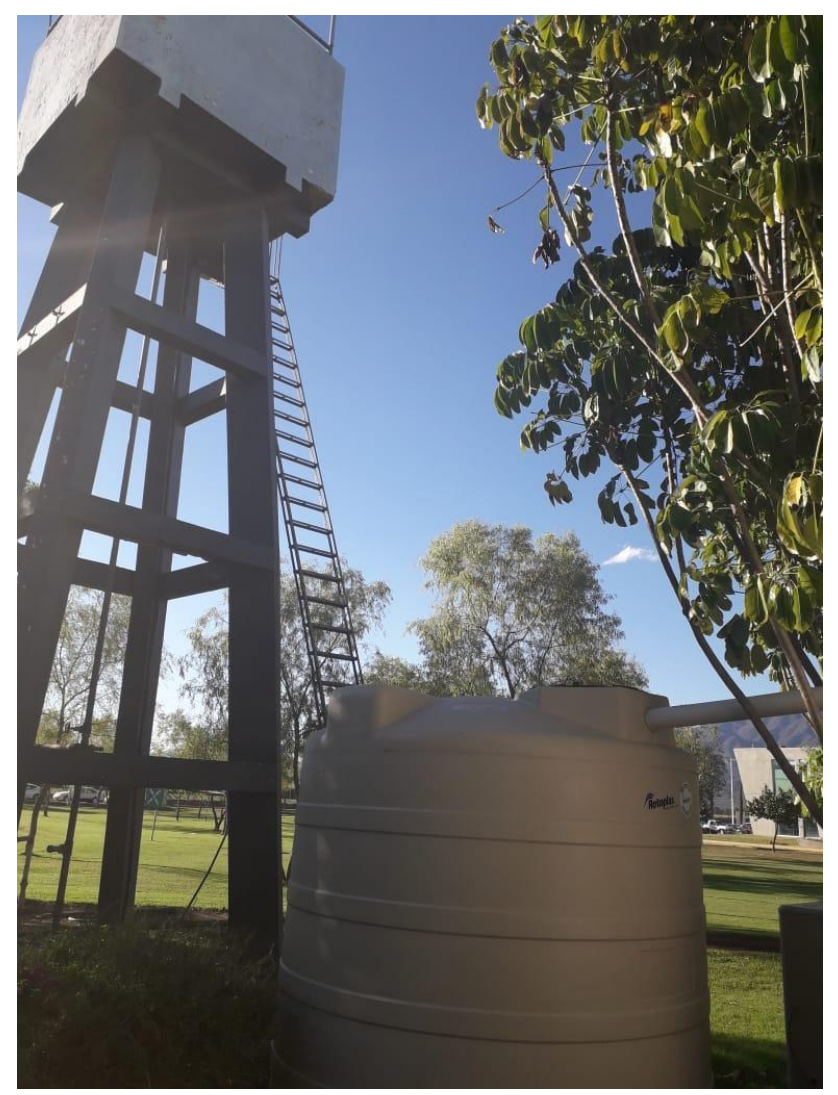

Figura 1 Sistema de captación de agua de lluvia instalado a un costado de la Rectoría del CUValles Fuente: Captado personalmente para el archivo de la presente investigación

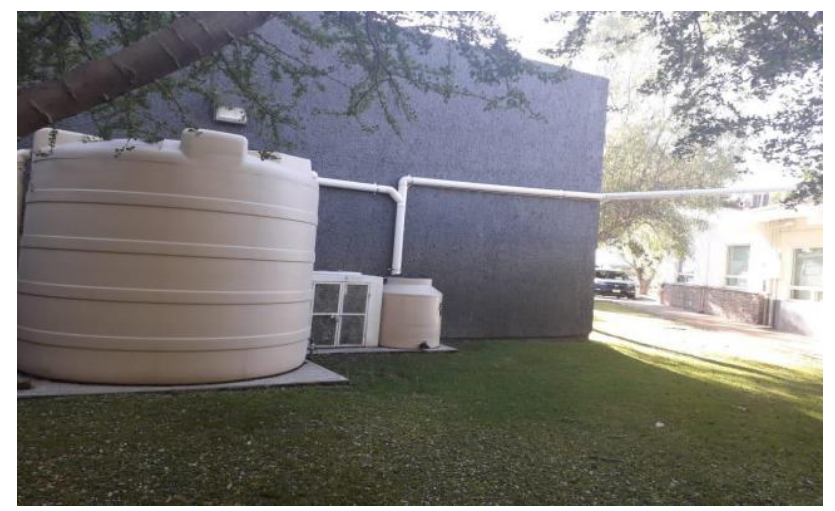

Figura 2 Sistema de Captación de Agua de lluvia, instalado a un costado del Área de Jurídicos del CUValles

Fuente: Captado personalmente para el archivo de la presente investigación

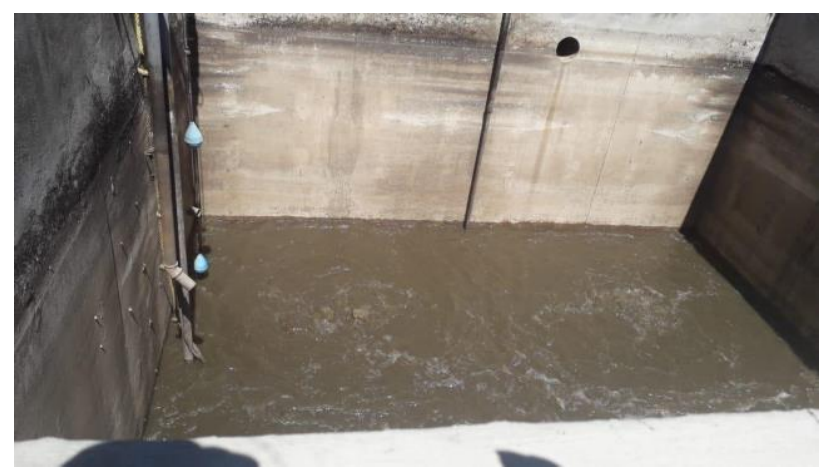

Figura 3 Planta de tratamiento de agua del CUValles Fuente: Captado personalmente para el archivo de la presente investigación

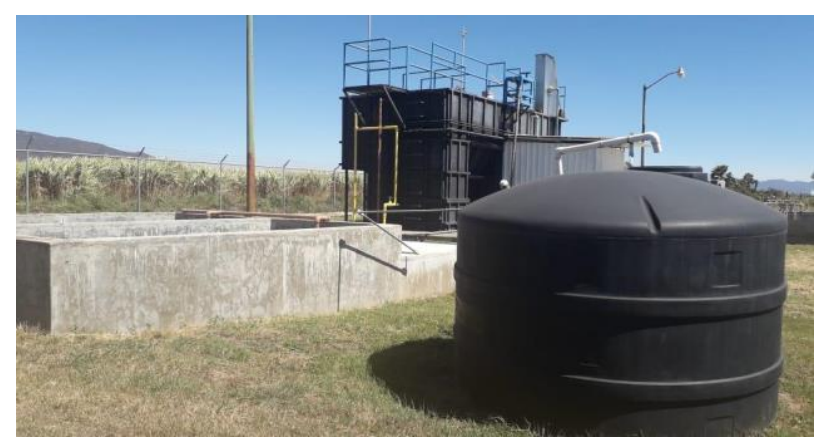

Figura 4 Infraestructura de la planta de tratamiento de agua del CUValles

Fuente: Captado personalmente para el archivo de la presente investigación

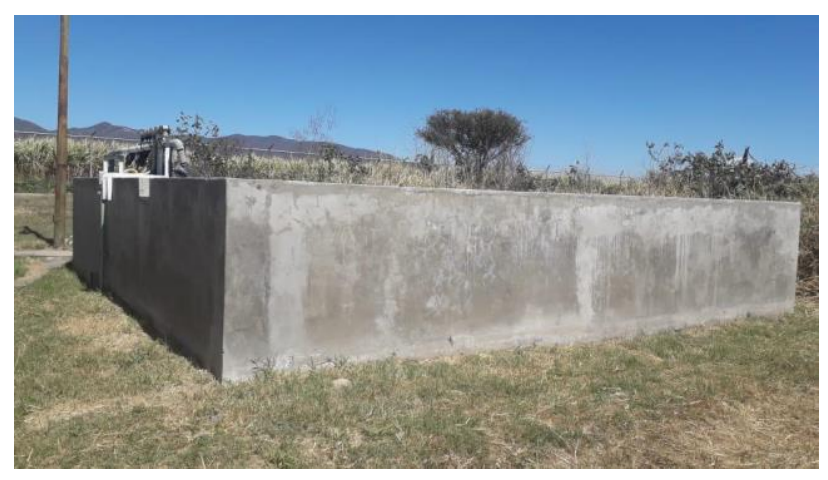

Figura 5 Infraestructura de la planta de tratamiento de agua del CUValles

Fuente: Captado personalmente para el archivo de la presente investigación

\section{Conclusiones}

En el actuar universitario existen áreas de oportunidad que deben ser potencializadas, uno de ellas es la formación profesional con valores de responsabilidad y compromiso con la sociedad y el medio ambiente, que generen propuestas de solución frente a los retos ambientales, procurando el uso responsable de los recursos financieros y naturales, estos últimos esenciales para la vida como el agua. 
Reforzando lo anterior y con base a las recomendaciones de organizaciones nacionales e internacionales, destaca la sensibilización a la comunidad universitaria a través de la educación formal e informal, en el contexto de la "educación para el desarrollo sostenible" aprovechando las herramientas que se tienen en las instituciones educativas en términos de capital humano en formación y profesionales en temas medioambientales, siendo quizá una solución simple, de aplicación inmediata y con resultados perdurables a largo plazo, al incluir los valores de responsabilidad social y ambiental y la nueva cultura del agua, con la ventaja de que puede ser difundida en toda la comunidad universitaria a través de la impartición periódica de cursos, talleres, foros, seminarios, conferencias, diplomados, contribuyendo al cuidado y uso racional de los recursos hídricos.

Otro aspecto relevante es la información, que debe ser plasmada en documentos, reportes, o sistemas de información que permitan la evaluación constante de indicadores y sirvan de base para fijar nuevas metas de acuerdo al contexto actual y futuro, garantizando así la mejora continua y que puedan ser replicados en otras Instituciones de Educación Superior.

Además de los ejes seleccionados para la investigación, existen otras áreas de oportunidad que se pueden trabajar para lograr un mayor impacto, incluso el CUValles pudiera ser acreedor al Reconocimiento como Líder Ambiental por parte de la Secretaría de Medio Ambiente y Desarrollo Territorial-SEMADET si logra la medición, documentación y consolidación de otras variables importantes que abonan a los indicadores de gestión ambiental estatal, como:

- $\quad$ Inspección y registro de fugas de agua.

- Sistemas de información para el reporte de fugas.

Medidores de gasto volumétrico.

- $\quad$ Registro del volumen de agua tratada.

- Títulos de concesión.

- $\quad$ Pagos de derecho, o en su caso convenio de aprovechamiento de agua.

Análisis de laboratorio para el control y monitoreo de las aguas residuales generadas en las instalaciones, siendo estos de los más significativos.

Consolidación de la Política ambiental.

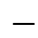

Involucramiento de la comunidad universitaria en el diseño de la política ambiental.

- Integración de Comités con presupuesto propio destinado a temas de sustentabilidad.

- Planes o Programas consolidados en materia hídrica.

Esta investigación se delimitó al recurso hídrico como variable principal, debido a la importancia y lo esencial que es para la vida. Si bien existen otros elementos que forman parte del medio ambiente y que es importante valorar cómo: la calidad del aire, el uso de los recursos energéticos, la gestión de residuos, entre otros, orientando acciones para minimizar el deterioro ambiental y contribuir a mejorar la calidad de vida, no así la investigación se acotó debido a la falta de información respecto a la medición y monitoreo de los factores antes mencionados, siendo una oportunidad para futuras líneas de investigación.

\section{Referencias}

ARROJO A. P (S.F.). La nueva cultura del agua del siglo XXI. Universidad de Zaragoza recuperado el 10 de febrero de 2019 de: https://www.zaragoza.es/contenidos/medioambi ente/cajaAzul/palabras/Arrojo_ES.pdf.

Asociación Española de Contabilidad y Administración de Empresas (2006). La semántica de la Responsabilidad Social Corporativa. Madrid. 1era. Edición. Editorial ORMAG. S.L.

Cámara de Diputados del H. Congreso de la Unión. (1992). Ley de Aguas Nacionales. México: Diario Oficial de la Federación. Recuperado el 10 de septiembre de 2018, de: http://www.diputados.gob.mx/LeyesBiblio/pdf/ 16_240316.pdf.

Carroll, A.B. (1979). A three-dimensional conceptual model of corporate performance. Academy of Management Review. 4(4) pp. 497-505. Recuperado el 20 de enero de 2019 de:

http://www.jstor.org/stable/10.2307/257850.

Comisión Mundial del Medio Ambiente y del Desarrollo (CMMAD). (1987). Informe Brundtland. Recuperado el 18 de mayo de 2019 de http://sds.uanl.mx/el-concepto-desarrollosustentable/.

ACEVES-MÁRQUEZ, Martha Patricia, AYALA-RAMIREZ, Suhey, CASTILLO-GIRON, Víctor Manuel y MEDINA-CELIS, Laura Margarita. Estrategias de gestión ambiental en las Instituciones de Educación Superior. El caso del Centro Universitario de los Valles. Revista del Desarrollo Urbano y Sustentable. 2019 
Castillo, V.M., Medina, L.M., Ayala, S. y Medina, G. (2017). Social Responsibility: a challenge for university management. Perú: Journal-Law and Economy, con Vol. 1 No. 1 e ISSN-On line: 2524-2113. Recuperado el 15 de marzo de 2019 de:http://www.rinoe.org/revistas/Journal_Law_ and_Economy/vol1num1/Journal_Law_and $\% 2$ 0Economy_V1_N1.pdf .

CONAGUA. (2017). Programa Nacional Hídrico (PNH) 2014-2018. Recuperado el 15 de octubre de 2018, de: https://www.gob.mx/conagua/acciones-y programas/programa-nacional-hidrico-pnh2014-2018\#documentos.

CUVALLES. (2018). Programas Educativos del CUValles recuperado el 10 de enero de 2019 de: http://cuvalles.udg.mx/oferta educativa.

Chavarro Velandia, A. (mayo de 2011). Claves de una gestión pública del recurso hídrico. Revista Gestión y Ambiente, 14(1), 7-22. Recuperado el 13 de septiembre de 2018, de: https://revistas.unal.edu.co/index.php/gestion/a rticle/view/25423/25945.

Centro Universitario de los Valles (CUValles). (2019a). Programas Educativos del CUValles recuperado el 10 de enero de 2019 de: http://cuvalles.udg.mx/oferta educativa.

-----CUVALLES. (2019b). Cuerpos Acdémicos. Recuperado el 20 de enero de 2019 de:

http://www.valles.udg.mx/investigacion/cuerpo s_academicos.

-----CUVALLES. (2019c). La Gaceta del Centro Universitario de los Valles. Recuperado el 20 de enero de 2019 de: https://issuu.com/gacetacuvallesoctubre2017/do cs/gaceta_enero_2019_2.

-----CUVALLES. (2019d). Misión y Visión. Universidad de Guadalajara. Recuperado el 20 de febrero de 2019 de: http://cuvalles.udg.mx/acerca_de/mision_vision

Comisión Mundial del Medio Ambiente y del Desarrollo. (CMMAD). (1987) Informe Brundtland. recuperado el 13 de mayo de 2019 de http://sds.uanl.mx/el-concepto-desarrollosustentable/.
MÜNCH L., ÁNGELES E. Métodos y Técnicas de Investigación. Editorial Trillas. 2015

Red de Indicadores de Sostenibilidad en las Universidades. (2014). Encuesta para el diagnóstico de la institucionalización del compromiso ambiental en las universidades colombianas recuperado el 22 de septiembre de 2018 de: file://C:/Users/VALLES_11/Downloads/201611

03_osz_encuesta_para_diagnstico\%20(1).pdf.

Organización de las Naciones Unidas (ONU). (2015). Agenda 2030 para el Desarrollo Sostenible. Recuperado el 23 de enero de 2019, de: http://www.onu.org.mx/agenda-2030/.

Tiburcio Sánchez, A., \& Perevochtchikova, M. (2012). La gestión del agua y el desarrollo de indicadores ambientales en México y Canadá: un análisis comparativo. Journal of Latin American Geography 2012, 11(2), 145-165. Recuperado el 7 de octubre de 2018, de: http://web.a.ebscohost.com.wdg.biblio.UDG.m $\mathrm{x}: 2048 /$ ehost/pdfviewer/pdfviewer?vid=5\&sid= e2de0884-32ab-4629-

9df66d7ca4cb5aec\%40sessionmgr4007.

Organización de las Naciones Unidas (ONU). (2015). Agenda 2030 para el Desarrollo Sostenible. Recuperado el 23 de enero de 2019, de: http://www.onu.org.mx/agenda-2030/.

Unidad de ecotecnologías. (S.F.) Sistema de Captación de Agua de Lluvia. Recuperado 10 de agosto de 2019 de http://ecotec.unam.mx/Ecotec/ecoteca/colectade-agua-pluvial-como-medida-para-elaprovechamiento-sustentable-de-la-energia.

Vallaeys, F. \& Álvarez Rodríguez. J. (2019). Hacia una definición latinoamericana de Responsabilidad Social Universitaria. Aproximación a las preferencias conceptuales de los Universitarios. Revista de la Facultad de Educación XX1. 22 (1). Recuperado el 20 de enero de 2019 de: doi:https://doi.org/10.5944/educxx1.19442.

Zapata-González, L, Quiceno-Hoyos, A. \& Tabares-Hidalgo, L. (2016). Campus universitario sustentable. Revista de Arquitectura. 18(2). 107-119. Recuperado el 20 de enero de 2019 de: doi:10.14718/RevArq.2016.18.2.10

ACEVES-MÁRQUEZ, Martha Patricia, AYALA-RAMIREZ, Suhey, CASTILLO-GIRON, Víctor Manuel y MEDINA-CELIS, Laura Margarita. Estrategias de gestión ambiental en las Instituciones de Educación Superior. El caso del Centro Universitario de los Valles. Revista del Desarrollo Urbano y Sustentable. 2019 